\title{
Una familia con síndrome de Wolfram
}

\author{
Gema Esteban Bueno ${ }^{a, b, c}$, Mónica Ruano García ${ }^{a, b}$, Sonia Grandes Velasco c,d, \\ Karina Villar Gómez de las Heras ${ }^{c, e, f}$
}

\begin{abstract}
a Unidad de Gestión Clínica Garrucha. Área de Gestión Sanitaria Norte de Almería (AGSNA). Almería (España).

${ }^{b}$ Equipo Multidisciplinar de Síndrome de Wolfram. AGSNA. Almería (España).

' Grupo semFYC de Genética Clínica - Enfermedades Raras. ${ }^{d}$ Centro de Salud Puerta Bonita. Dirección Asistencial Centro. Servicio Madrileño de Salud. Madrid (España).

e Dirección General de Atención Sanitaria y Calidad. Servicio de Salud de Castilla-La Mancha. Toledo (España).

${ }^{\dagger}$ Alianza Española de Familias de von Hippel-Lindau.
\end{abstract}

Correspondencia: Gema Esteban Bueno. Correo electrónico: aswolfram@hotmail. com

Recibido el 20 de mayo de 2013.

Aceptado para su publicación el 3 de abril de 2013.

\begin{abstract}
RESUMEN
El Síndrome de Wolfram o DIDMOAD es una compleja entidad cuyos principales componentes son diabetes mellitus, diabetes insípida, atrofia óptica y sordera. Además se suelen asociar otras alteraciones en frecuencia variable. Es una patología considerada rara por su baja prevalencia y sus repercusiones psicosociales. Se manifiesta durante la infancia siendo de carácter degenerativo y progresivo. Se asocia generalmente a trasmisión autosómica recesiva.

Como ejemplo de este síndrome presentamos el caso de una familia compuesta por tres hermanos afectos.
\end{abstract}

PALABRAS CLAVE: Síndrome de Wolfram. Atrofia Óptica. Diabetes Mellitus. Enfermedades Raras.

\section{ABSTRACT}

Wolfram syndrome or DIDMOAD is a complex condition whose main components are diabetes mellitus, diabetes insipidus, optic atrophy and deafness. Other problems are also associated with varying frequency. It is a pathology which is considered to be rare due to its low prevalence and psycho-social repercussions. It occurs during childhood and is degenerative and progressive. It is generally associated with autosomal recessive transmission. As an example of this syndrome, we report the case of a family of three affected siblings.

KEYWORDS: Wolfram syndrome. Optic atrophy. Diabetes Mellitus. Rare diseases.

\section{NTRODUCCIÓN}

El Síndrome de Wolfram (SW) es una rara y compleja enfermedad neurodegenerativa progresiva, de transmisión autosómica recesiva (4p) y excepcionalmente de herencia mitocondrial o esporádica. Fue descrito en 1938 por el Dr. J.Wolfram al observar la enfermedad en cuatro de ocho hermanos, que presentaban diabetes mellitus y atrofia óptica' ${ }^{1}$. Se ha estimado que la prevalencia en Estados Unidos es 1/100.000 habitantes y en Reino Unido de 1/770.000. En España es desconocida. La frecuencia de portadores es de $1 / 354^{2}$. Existe consanguinidad en los padres en un $27 \%$ de los casos $^{3}$.

El SW también se conoce por el acrónimo DIDMOAD, que corresponde a las iniciales en inglés de sus componentes principales: diabetes insípida (DI, presente en un $62 \%$ de los pacientes), diabetes mellitus (DM), atrofia óptica (OA) y sordera neurosensorial (D,73\%). En algunos casos se suman alteraciones del tracto urinario (58\%), sistema nervioso central $(62 \%)$ y atrofia gonadal ${ }^{4}$. Algunos autores han sugerido la denominación DIDMOADUA, dada la alta frecuencia de las alteraciones urinarias (tabla 1).

La historia natural del síndrome sugiere que casi todos los pacientes desarrollarán la mayoría de las complicaciones de la enfermedad ${ }^{5}$, por lo que el diagnóstico del SW suele implicar devastadoras consecuencias físicas y emocionales. El fallecimiento suele producirse en la tercera o cuarta década de la vida ${ }^{4,6}$.

\section{OBSERVACIONES CLÍNICAS}

Hablamos de tres hermanos de una misma familia sin antecedentes de interés, niños sanos al nacimiento. Antecedentes familiares: sordera en familiares de segundo grado. 


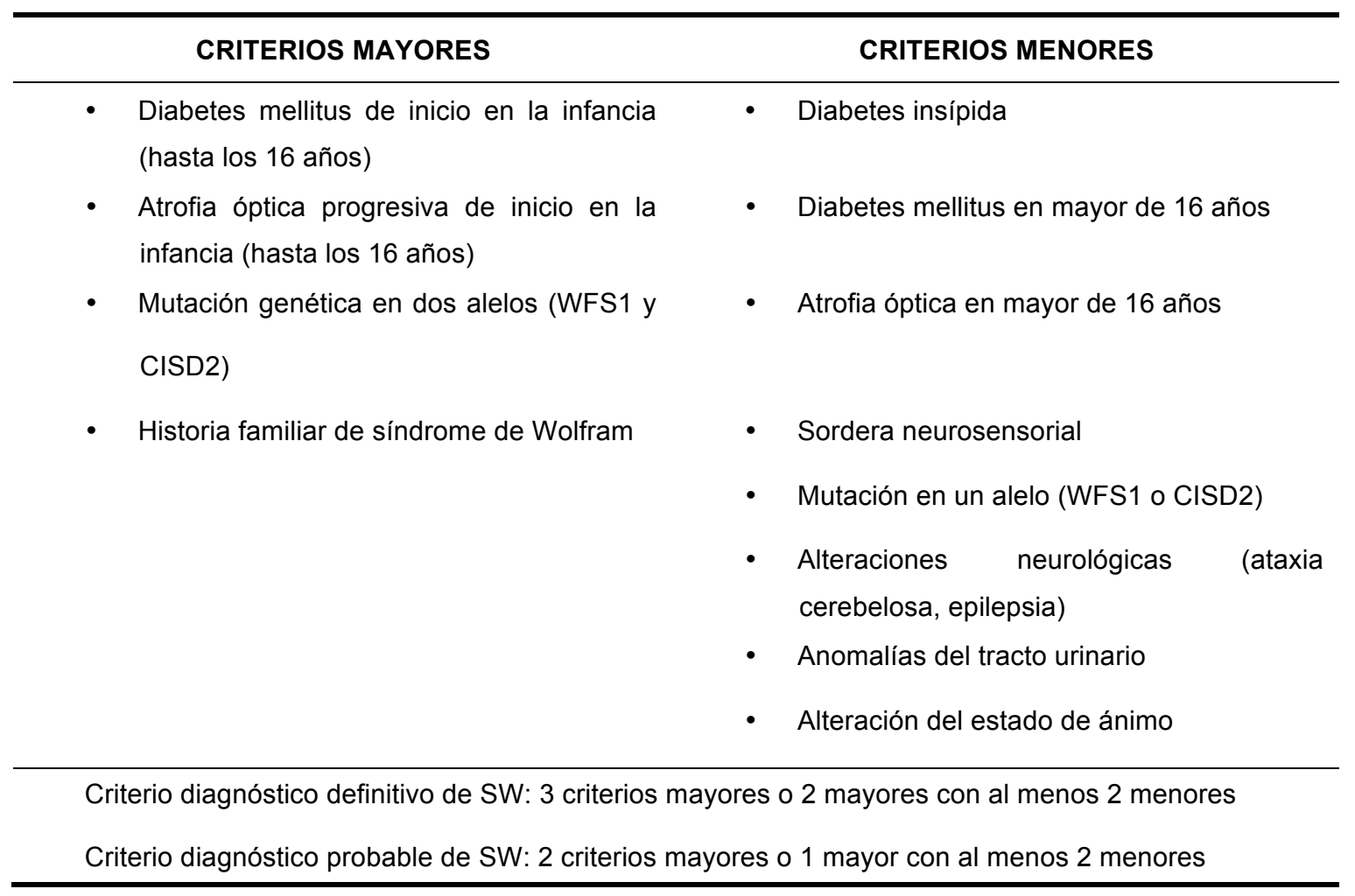

Tabla 1. Criterios diagnósticos del síndrome de Wolfram (SW).

El hermano mayor, a la edad de 3 años comienza con DM de inicio insidioso, con glucemias elevadas y sin cetosis, requiriendo insulina para su control. Un año después aparecen los problemas de visión, achacándose a problemas de refracción. No es diagnosticado de atrofia del nervio óptico (AO) hasta los 6 años. Tras múltiples consultas que incluían especialistas privados, se diagnostica erróneamente de neuropatía óptica hereditaria de Leber. La ausencia de respuesta a los tratamientos lleva a la familia a continuar consultando a especialistas de diferentes lugares. Finalmente es diagnosticado de SW a los 14 años de edad. Poco a poco, a partir de esta edad se aprecia atrofia gonadal con elevación de FSH y LH y testosterona disminuida, recibiendotratamientocontestosterona. A partir de los 15 años aparece la sordera bilateral con caída de frecuencias agudas. A los 16 años presenta ya ceguera completa y, alrededor de los 20 años, cocleopatía degenerativa bilateral con afectación del equilibrio. La presencia de vejiga neurogénica le obliga a realización de sondajes urinarios. La ecografía renal muestra hidronefrosis bilateral con datos analíticos de insuficiencia renal. Con 16 años aparecen los primeros síntomas de $\mathrm{DI}$, que se trata con desmopresina, con buen control. No es visto por el neurólogo hasta los 30 años, encontrándose entonces en las pruebas de imagen una atrofia pontocerebelosa. Otros síntomas que se irán presentando son: anosmia, ataxia, sudoración profusa y alteración en la regulación de la temperatura corporal, insomnio, ageusia, hipertensión arterial. Fallece con 42 años por insuficiencia renal y sus complicaciones.

Los síntomas de DM comienzan a aparecer en los hermanos en edades más tardías, con 6 y 8 años de edad respectivamente. Progresivamente van apareciendo el resto de alteraciones típicas del SW que fue presentando el hermano mayor: $\mathrm{AO}, \mathrm{DI}$, sordera, vejiga neurogénica. Con una evolución similar, fallecen con 38 y 37 años de edad por insuficiencia renal y fallo multiorgánico (tabla 2). Sus limitaciones físicas no impidieron que realizaran estudios universitarios, pues no existe en el SW afectación en el desarrollo intelectual.

El estudio genético se realizó ya avanzada la enfermedad (el hermano mayor tenía 30 años), no con fines de confirmación diagnóstica, sino para consejo genético de familiares/pacientes y evitar su transmisión a lo largo y ancho del árbol familiar. 


\begin{tabular}{lccccc}
\hline & DM & AO & DI & Sordera & Vejiga neurogénica \\
Hermano mayor & 3 & 6 & 16 & 15 & 15 \\
Hermano mediano & 6 & 9 & 11 & 20 & 20 \\
Hermano pequeño & 9 & 9,5 & 13 & 11 & 11 \\
\hline
\end{tabular}

Tabla 2. Edad de aparición de los síntomas en los tres hermanos. DM: Diabetes mellitus. AO: Atrofia óptica. DI: Diabetes insípida.

\section{COMENTARIOS}

El médico ha de sospechar este síndrome ante un paciente con DM de inicio en la edad pediátrica a la que se le asocie $A^{5,7}$, tras descartar otras patologías que pueden incluir estas dos entidades (síndrome de la rubéola congénita, neuropatía óptica hereditaria de Leber, síndrome anémico tiamina dependiente, ataxia de Friedreich).

En caso de tener un hermano afectado por este síndrome, sólo la presentación de DM en otro hermano justificaría una alta sospecha de SW. Así, en el caso presentado debía de haberse sospechado en los otros dos hermanos y haber realizado el diagnóstico genético confirmatorio mucho antes.

La DM tipo 1 se presenta en la primera década de la vida (edad media de 6 años) y suele ser la manifestación más temprana de la enfermedad ${ }^{8}$. Es insulinodependiente, no autoinmune, con menor prevalencia de cetoacidosis, y la presencia de complicaciones microvasculares es poco frecuente incluso tras 25 años de evolución ${ }^{3}$. Se suele seguir de $\mathrm{AO}$, que aparece en la segunda década (en torno a los 11 años) con pérdida progresiva de la agudeza visual y la visión del color (discromatopsia), y desembocando en ceguera en la edad adulta. La DM en edad pediátrica asociada a la $A O$ es criterio diagnóstico de SW, presentándose casi en el $100 \%$ de los casos. Se puede realizar diagnóstico presuntivo en un niño que presente simultáneamente DM tipo 1 y $\mathrm{AO}$, tras descartar otras patologías que incluyan AO o DM (como la neuropatía óptica hereditaria de Leber) ${ }^{9}$.

La sordera neurosensorial es bilateral, simétrica y progresiva, se inicia en la segunda década (en torno a los 16 años) con pérdida de las frecuencias altas. La DI central suele aparecer en torno a los 14 años.

Las anomalías del tracto urinario aparecen en la tercera década (en torno a los 20 años), con atonía vesical, incontinencia e infecciones de repetición. La vejiga neurogénica parece ser secundaria a una neuropatía selectiva autonómica y puede favorecer las infecciones renales retrógradas, dañando seriamente el riñón. El diagnostico precoz y riguroso de estas manifestaciones puede mejorar significativamente la calidad de vida y las posibilidades de supervivencia ${ }^{5}$.

Las complicaciones neurológicas suelen aparecer en la cuarta década de la vida (en torno a los 35 años), siendo las más características ataxia, nistagmo horizontal, disartria, etc ${ }^{2}$. También son frecuentes el hipogonadismo hipergonadotrofo (más frecuente en hombres) y los trastornos psiquiátricos y del sueño ${ }^{8}$.

Aunque actualmente no existe tratamiento curativo, es fundamental un diagnóstico precoz y un seguimiento adecuado de los pacientes. El tratamiento temprano de las entidades que conforman el SW es fundamental para prevenir las complicaciones, mantener la mejor calidad de vida posible y evitar mortalidad prematura.

La DM precisa tratamiento insulínico, la DI la administración de desmopresina, la sordera neurogénica precisa de audífonos e implantes cocleares y la $\mathrm{AO}$ se beneficiará de las lentes de aumento hasta la pérdida total de visión. Respecto a la vejiga neurogénica, no todos responden igual a los anticolinérgicos y finalmente la mayoría precisan recurrir a los autosondajes intermitentes o sondajes permanentes. Para el hipogonadismo precisarán terapia hormonal sexual sustitutiva. Dado que es una enfermedad neurodegenerativa se recomienda la toma de coenzima $Q$ y complejos de vitamina $B$, aun cuando su efectividad no ha sido contrastada.

Hay pocos estudios sobre la edad de fallecimiento, que suele ser inferior a 50 años, generalmente por fallo respiratorio central con atrofia del troncoencéfalo, o por fallo renal ${ }^{4}$. Por ello es fundamental un adecuado $y$ buen control 
nefrourológico.

Este tipo de patologías crónicas progresivas y de inicio en la infancia requieren valoración también en el plano psicológico y social tanto del afectado como de la familia ${ }^{10}$.

Según hemos visto, el seguimiento y tratamiento debe ser multidisciplinar ${ }^{4,6}$, precisando de la coordinación del médico de familia o pediatra con el endocrinólogo en primer lugar, y posteriormente con oftalmólogo, otorrinolaringólogo, urólogo, internista, genetista, neurólogo, psiquiatra y psicólogo.

Desde el año 2011 existe un equipo médico multidisciplinar en España (Hospital La Inmaculada, Huercal-Overa, Almería) que realiza el seguimiento integral de estos pacientes. Este equipo está coordinado por un médico de familia, quien fundó, junto a la familia que hemos presentado como caso clínico hace ya 12 años, la Asociación del Síndrome de Wolfram. La mejora en el seguimiento ha producido un descenso en la mortalidad por insuficiencia renal, siendo actualmente el fallo del sistema nervioso central la principal causa de fallecimiento.

\section{COMENTARIOS}

Queremos agradecer tanto a la Asociación Española para la Investigación y Ayuda al Síndrome de Wolfram como a los afectados su inestimable colaboración. Sin ellos no hubiera sido posible mejorar el conocimiento de esta enfermedad.

\section{BIBLIOGRAFÍA}

1. Wolfram DJ, Wagener HP. Diabetes mellitus and simple optic atrophy among siblings: report of four cases. Mayo Clin Proc. 1938; 13: 715-8.

2. Barrett TG, Bundey SE, Macleod AF. Neurodegeneration and diabetes: UK nationwide study of Wolfram (DIDMOAD) syndrome. Lancet. 1995; 346: 1458-63.

3. Lou Frances G, Soto de Ruiz S, Lopez-Madrazo Hernández MJ, Macipe Costa R, Rodriguez Rigual M. Sindrome de Wolfram. Estudio clinico y genetico en dos familias. An Pediatr (Barc). 2008; 68 (1): 54-7.

4. Pickett KA, Duncan RP, Paciorkowski AR, Permutt MA, Marshall B, Hershey T et al; Washington University Wolfram Study Group. Balance impairment in individuals with Wolfram syndrome. Gait Posture. 2012; 36 (3): 619-24.

5. Kumar S. Wolfram syndrome: important implications for pediatricians and pediatric endocrinologists. Pediatr Diabetes. 2010; 11 (1): 28-37.

6. Marshall BA, Permutt MA, Paciorkowski AR, Hoekel J, Karzon R, Wasson $\mathrm{J}$ et al; Washington University Wolfram Study Group. Phenotypic characteristics of early Wolfram syndrome. Orphanet J Rare Dis. 2013; 8: 64.

7. Poswar FO, Carneiro JA, Alves IM, Oliveira Junior ER, Dias LT et al. Consanguinidade e síndrome de Wolfram. Relato de caso. Rev Soc Bras Clin Med. 2012; 10 (2): 155-7.

8. Rohayem J, Ehlers C, Wiedemann B, Holl R, Oexle K, Kordonouri $\mathrm{O}$ et al. Diabetes and neurodegeneration in Wolfram syndrome: a multicenter study of phenotype and genotype. Diabetes Care. 2011; 34 (7): 1503-10.

9. Ganie MA, Bhat D. Current developments in Wolfram síndrome.J Pediatr Endocrinol Metab. 2009; 22 (1): 3-10.

10. Vilan A, Faria O, Campos MM. Síndrome de Wolfram. Rev Neurol 2009; 49 (4): 221-2. 